\title{
Kinetics of color development in glucose/Amino Acid model systems at different temperatures
}

\author{
Ana Paola Echavarría 1, 2, *; Jordi Pagán ${ }^{2}$; Albert Ibarz \\ ${ }^{1}$ Facultad de Ciencias de la Salud, Universidad Estatal de Milagro (UNEMI), Cdla. Universitaria Km. 1.5 vía Milagro \\ Km. 26, Guayas, Ecuador. \\ 2 Departamento de Tecnología de Alimentos, Universidad de Lleida, Av. Rovira Roure, 191, 25198 Lleida, España, \\ teléfono: (+34) 973 702817, fax.: (+34) 973702596.
}

Received August 22, 2015. Accepted January 20, 2016.

\begin{abstract}
This study investigated the influence of temperature on the color development of melanoidins formed from a single combination of glucose with amino acid. The selected amino acid, commonly found in apple juice and highly reactive in the Maillard reaction, were asparagine (Asn), aspartic acid (Asp) and glutamic acid (Glu). For this, the color development was evaluated by measuring browning at $420 \mathrm{~nm}$ and color measurements by spectrophotometry and colorimetry methods. The effect of temperature on the color intensity, the absorption of melanoidins were also measured at different wavelengths $(280,325,405)$. The value of melanoidins formed from all model systems was located on a dominant wavelength of $325 \mathrm{~nm}$, the ultra violet zone of the diagram. A first-order kinetic model was applied to $L^{*}$ and the evolution of color difference $\Delta E^{*}$. In addition, $\mathrm{a}^{*}, \mathrm{~b}^{*}$ values, significantly differences were found in the glucose/aspartic acid model system in the brown-red zone. Therefore, the color development of the melanoidins was influenced by the type of amino acid and temperature, and it is thought that the $\mathrm{a}^{*}$ and $\mathrm{b}^{*}$ values can be used to explain the differences among the amino acid in the color development of melanoidins.
\end{abstract}

Keywords: antioxidant activity; phenolic compounds; residue flour; DPPH assay.

\section{Introduction}

The first quality impact by which the consumers take the decision to acquire a product is its visual appearance. The color of products and various reactions such as pigment destruction (carotenoids and chlorophylls) and non-enzymatic browning (Maillard) reactions, can occur during heating of fruits and vegetables and therefore affect its color (Ganjloo et al., 2009). The retention of total color can be used as a quality indicator to evaluate the extent of deterioration due to thermal processing (Demirhan and Özbek 2009).

Several researchers have published work on modelling of thermal degradation kinetics of color in the temperature range of sterilization conditions (Rattanathanalerk et al., 2005). In model systems reducing sugars or Maillard intermediates were used as starting materials and defined colored reaction products could be isolated and identified (Rufián-Henares and Morales, 2007). Also it is known that many products especially fruits darken during storage, it is attributed mainly to nonenzymatic reactions (Bharate et al., 2014). These reactions involve Maillard reaction and ascorbic acid degradation (occurs by an oxidative path in citrus juices) (Kim et al., 2004). The Maillard reaction, taking place between amino groups and reducing sugars, is the most important cause of browning in apple juice (Echavarría et al., 2011). Maillard browning may be desirable during food processing, as in the manufacture of coffee, tea, beer and in the toasting and baking of bread. This reaction improves desirable sensory characteristics of these foods e.g.

\footnotetext{
* Corresponding author

E-mail: echavarria @tecal.udl.cat (A.P. Echavarría).
} 
color, aroma and flavour (Jaeger et al., 2010).

The Maillard reaction development is generally monitored by the increase in absorbance either 280 early Maillard Reaction Products (MRPs) for pyrazine compounds (Gu et al., 2010), 320-350 (soluble pre-melanoidins, advanced stage) or 420-450 $\mathrm{nm}$ (final MRPs), corresponding to color intensity of the reaction medium, as well as the formation of specific compounds (Billaud et al., 2004). Browning in juices and fruit purees during manufacture and storage is of vital interest for the industry. Different indicators were assayed to determine the extent of browning (Echavarría et al., 2012). These included absorbance measurements at given wavelengths, being $420 \mathrm{~nm}$, one of the most frequently used for juices, as well as colorimetric evaluations and qualitative and quantitative determinations of intermediate and final products, such as sugars and, especially, hydroxymethylfurfural (HMF). Generally, the low absorbance values recorded at 420 $\mathrm{nm}$ seemed to reveal a less proportion in brown pigments or melanoidins (Echavarría et al., 2013). Reducing sugars presents in the fruit puree, mainly glucose and fructose, participate directly in the nonenzymatic browning reactions. Some disaccharides, such as sucrose, can also hydrolyze during thermal treatment, leading to glucose and fructose formation. In consequence, the evolution of sugar content can be used as an indicator of nonenzymatic browning variation and has been studied by some authors (Valdramidis et al., 2010; Contreras-Calderón et al., 2009).

The color of products can be specified by three co-ordinates in the color space which can be obtained directly with a tristimulus colorimeter. The $L^{*}, \mathrm{a}^{*}$ and $\mathrm{b}^{*}$ system is the more frequently used scale to measure the color of food products (Zyzelewicz et al., 2014). The $L^{*}$ value is a measure of the lightness, the $b^{*}$ value indicates the change of the color from blue to yellow, and the $\mathrm{a}^{*}$ value the change from green to red (Kim and Lee 2008).

The main objective of this work was to determinate the influence of temperature on the color development of melanoidins formed from glucose with a single combination of amino acid (Asn, Asp or Glu) model systems, using different conditions: heating time, temperature and amino acid concentration. The UVabsorbance $\left(\mathrm{A}_{280}, \mathrm{~A}_{325}\right.$ and browning at $\left.\mathrm{A}_{420} \mathrm{~nm}\right)$ were also examined.

\section{Material and methods}

\subsection{Chemicals and reagents}

D-Glucose, L-Asparagine, Glutamic acid and Aspartic acid, Sodium Hydroxide $(0.25 \mathrm{~N})$ were purchased from (Panreac Química S.A.U, Barcelona,Spain), pyridine (Merck, Hohenbrunn, Germany), active carbon (Probus, S.A. Barcelona, Spain). Deionized and distilled water were used throughout the research.

\subsection{Methods}

Three model systems were prepared from a single combination of glucose $(\mathrm{G})$ and amino acid (Asn, Glu, Asp). Water-soluble melanoidins were obtained from different Maillard model systems by dissolving $50 \mathrm{~g}$ of each sugar with $0.7 \mathrm{~g} / \mathrm{kg}$ of amino acid in $250 \mathrm{~mL}$ of distilled water. This solution in a sealed container was treated a 96 hours in a stove at different temperature $(50,85$, $120^{\circ} \mathrm{C}$ ). Melanoidins formed during the thermal treatment were isolated as described by Ibarz et al. (2008). The melanoidins extracted were lyophilized (Cryodos-50/230V-50 Hz Telstar, Madrid, Spain).

\subsection{Analytical determinations}

After measuring the soluble solid content of each sample with an Atago RX-1000 digital refractometer, they were diluted to $12{ }^{\circ}$ Brix with doubly distilled water according to Carabasa-Giribet and IbarzRibas (2000). The $\mathrm{pH}$ of the model melanoidins was determined with a LabBasic 20 (Crison. Barcelona, Spain) pH- 
meter, sample was adjusted to 7.5 with 1 $\mathrm{mol} / \mathrm{L} \mathrm{NaOH}$.

\subsection{UV-absorbance and browning intensity}

The UV-absorbance of the melanoidins fraction was determined with absorbance spectrum detection between $280 \mathrm{~nm}$ to 420 $\mathrm{nm}$ using a Helios gamma spectrophotometer (Thermo Fisher Scientific Inc., Waltham, MA, USA). Using a $1 \mathrm{~cm}$-path length cell. Samples were dissolved in deionized water at a concentration of 0.1 $\mathrm{mg} / \mathrm{mL}$.

\subsection{Color determination by optimum conditions}

The color of melanoidins fractionations were directly measured using the CIE (Commission Internationale de l'Eclairage) values $L^{*}$ (lightness), $\mathrm{a}^{*}$ (redness), $\mathrm{b}^{*}$ (yellowness) and $\Delta \mathrm{E}^{*}$ color difference, which is expressed as Equation (1).

$\Delta \mathrm{E}^{*}=\sqrt{\left.\left(\Delta a^{*}\right)^{2}+\left(\Delta b^{*}\right)^{2}+\left(\Delta L^{*}\right)^{2}\right]} \quad$ (Eq. 1)

These values were determined with a light source C, with a color-meter (Minolta ChromaMeter Model CR-400, Konica Minolta, Tokyo, Japan). The equipment was set up for illuminant D65 and $10^{\circ}$ observer angle and calibrated using a standard white reflector plate.

\subsection{Kinetic considerations}

The complexity of fruit by-products gives rise to a wide range of non-enzymatic browning reactions during their thermal treatment. Consequently, it is difficult to establish a reaction mechanism and to obtain a kinetic model that adequately describes the whole process. The model system is commonly used to determinate the color analysis. The data were subjected to regression analysis. Firstorder and pseudo-zero-order kinetic models have been used to evaluate the development of non-enzymatic browning (Ibarz et al., 2000). These kinetic models are expressed by the equations:

zero-order: $C=C_{0}+k_{0} t$ first-order: $C=C_{0} \exp \left(-k_{1} t\right) \quad$ (Eq. 3) where $\mathrm{C}$ is the value of the variable studied at time $\mathrm{t}, \mathrm{C}_{0}$ is the value of the variable studied at the initial time $\left(\mathrm{t}_{0}\right), \mathrm{k}_{0}$ is the zero-order kinetic constant and $\mathrm{k}_{1}$ is the first-order kinetic constant.

This two-stage kinetic mechanism can be expressed mathematically. If $\mathrm{C}$ is the variable used to measure the color changes caused by non-enzymatic browning reactions, then

$\frac{d C}{d t}=k_{0}-k_{1} C$

This equation can be integrated $\left(\mathrm{C}=\mathrm{C}_{0}\right.$ at $t=t_{0}$ ) to give

$C=K-\left(K-C_{0}\right) \exp \left(-k_{t} t\right)$

$K=\frac{k_{0}}{k_{1}}$

Also the fractional conversion model can be used to describe the color degradation (Ibarz et al., 2010).

$\frac{C-C_{f}}{C_{0}-C_{f}}=\exp (-k t)$

Where $C_{f}$ is the final equilibrium value of the color parameter

\subsection{Statistical analysis}

Data are presented as mean \pm standard deviation (SD). Differences between the mean values for individual combination of aminoacid were analyzed by variance (ANOVA) with Duncan's multiple range test. $\mathrm{p}<0.05$ was considered to indicate a statistically significant difference. Duncan's multiple range test using the $\begin{array}{llll}\text { OriginPro } & \text { 8SRO V8.0724 (B724) }\end{array}$ statistical analysis system (Origin-Lab Corporation, 2007. Northampton U.S.A) was used for statistical analyses.

\section{Results and discussion}

\subsection{Browning and formation of melanoidins}

Figure 1 shown the browning development of melanoidins from G/Asn at different time $\left(0,60\right.$ and 120 hours) at $120{ }^{\circ} \mathrm{C}$. The final stage of the browning reaction was 
monitored by the increase in absorbance at $420 \mathrm{~nm}$. In the present study, browning development was increased as increasing temperature treatment for all glucose and amino acids (Asn, Asp and GGlu) combinations. Except for GAsp model system that show different absorption in the UV-vis spectra $(375 \mathrm{~nm})$.

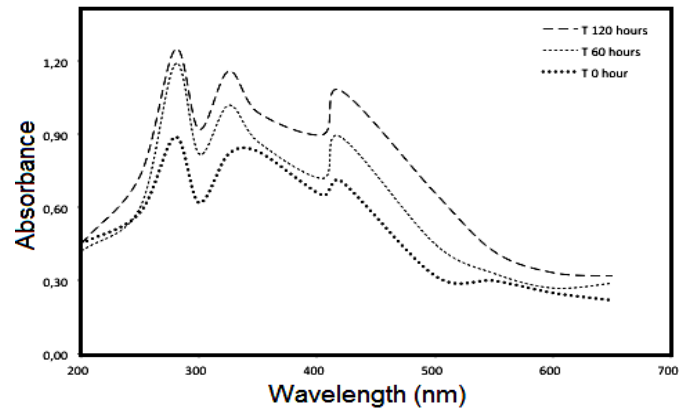

Figure 1. UV-vis spectrum at different time of melanoidins formed from glucose/Asn.

Every peak has a stable absorbance appeared in the range between $280 \mathrm{~nm}$ and $325 \mathrm{~nm}$, characteristic of melanoidins (Fig. 1). The highest absorption is formed in GAsn. After crossing the UV region, the absorption curve progressively came into the blue-absorbing region of the visible spectrum and yellow- brown colors appeared. The compounds formed early in the Maillard reaction absorb in the UV (Kim and Lee, 2008).

\subsection{Evolution of color parameters}

One of the best parameters for describing color variation is the color difference $\Delta E^{*}$, since it is a combination of the parameters $L^{*}, a^{*}$ and $b^{*}$. The evolution of $\Delta E^{*}$ with treatment time at different temperatures are shown at $50{ }^{\circ} \mathrm{C}$ (Fig. 2a), $85^{\circ} \mathrm{C}$ (Fig. 2b) and $120^{\circ} \mathrm{C}$ (Fig. 2c). In these case, all the systems shown the same behavior, reaching a maximum about 60 minutes and a plateau at prolonged time. In the Fig. 2c GAsn increased with time. Eq. 7 was used to fit the data to a kinetic model, with the color parameter $\mathrm{C}$ replaced by $\Delta E^{*}$. To obtain this value, the color of the untreated sample was used as a reference. Thus $\Delta E_{0}^{*}$ (the initial value) is zero and Eq. 5 (Ibarz et al., 2000) becomes:

$\Delta E^{*}=K-K_{0} \mathrm{e}^{(-k, t)}$
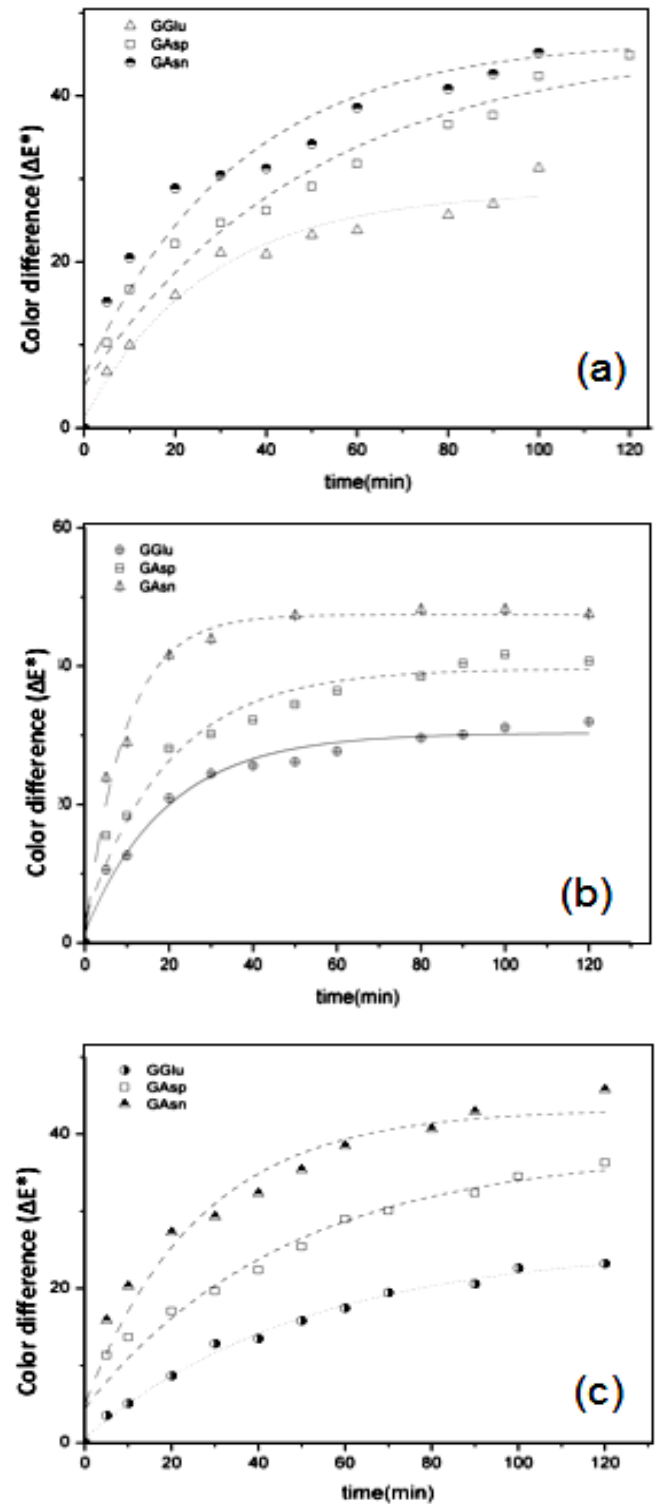

Figure 2. Color difference $\left(\Delta E^{*}\right)$ evolution for glucose and amino acid (Asn, Asp and Glu) model system with time and temperature of treatment at a) $50{ }^{\circ} \mathrm{C}$, b) $85^{\circ} \mathrm{C}$ and c) $100{ }^{\circ} \mathrm{C}$.

Where the color difference is $\Delta E^{*}=K$ when $t \rightarrow \infty$ and $\Delta E^{*}=K-K_{0}$ when $t \rightarrow 0, k_{l}$ is a reaction constant and $\mathrm{A}$ is the maximum color difference value reached at every temperature during the interval of heating studied. Calculated values of $A$ and $k_{l}$ for model systems at a different temperature are summarized in Table 1.

Data from Table 1 were fitted to Eq. (7) to give the various kinetic parameters of this equation; the results were significant at 95\% probability level. 
Table 1

Parameters of changes $\left(\Delta E^{*}\right)$ of color in the melanoidin model system

\begin{tabular}{lcccc}
\hline Melanoidins & $\begin{array}{c}\mathrm{T} \\
\left({ }^{\circ} \mathrm{C}\right)\end{array}$ & $K$ & $\begin{array}{c}k_{1} \times 10^{3} \\
\left(\mathrm{~min}^{-1}\right)\end{array}$ & $\mathrm{R}^{2}$ \\
\hline \multirow{2}{*}{ GAsn } & 50 & 33.58 & 4.89 & 0.920 \\
& 85 & 30.32 & 2.35 & 0.984 \\
& 120 & 25.46 & 0.97 & 0.944 \\
\hline \multirow{2}{*}{ GAsp } & 50 & 49.44 & 3.98 & 0.948 \\
& 85 & 39.60 & 3.10 & 0.961 \\
& 120 & 37.81 & 2.57 & 0.956 \\
\hline \multirow{3}{*}{ GGlu } & 50 & 28.54 & 3.71 & 0.964 \\
& 85 & 47.41 & 2.08 & 0.978 \\
& 120 & 48.83 & 1.19 & 0.993 \\
\hline
\end{tabular}

In order to make a better estimate of the kinetic parameters, a non-linear regression was applied to all data (Vaikousi et al., 2008).
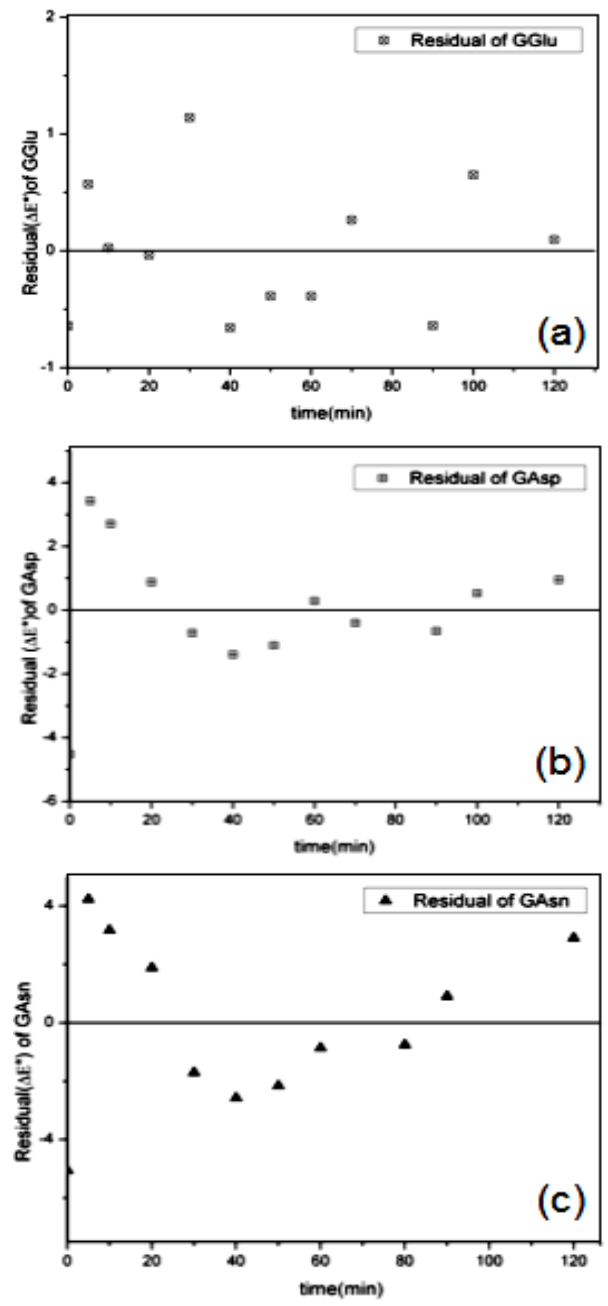

Figure 3. Plot of residuals for the $\Delta E^{*}$ parameter regression of the first order kinetic model as a function of the independent variable $(t)$.
Based on the examination of the residuals, these proved that the first-order kinetic model to be the most adequate, once the distribution of residuals has no visual tendency (were randomly distributed around zero). One example of these plots is showed in Fig. 3 for the $\Delta E^{*}$ parameter. On the other hand, an increase in treatment temperature and time caused a darkening of the model solution.

This is reflected in the decrease of lightness. In Figure 4 is shown for the treatment time of glucose and amino acid (Asn, Glu and Asp) model solution at different temperatures and the decrease lines fitted firstorder kinetic reaction throughout the heating period, this decree-sing value indicates that the samples were turned darker.
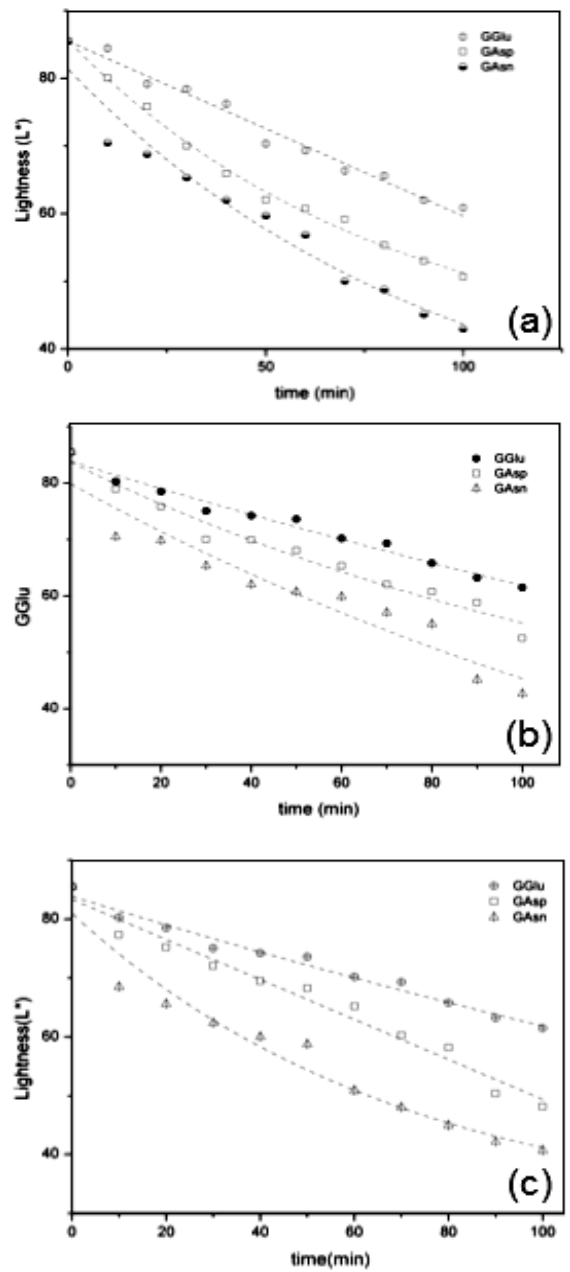

Figure 4. Evolution of $L^{*}$ for the glucose and amino acid (Asn, Asp and Glu) model system(a) at $50{ }^{\circ} \mathrm{C}$, (b) at $85^{\circ} \mathrm{C}$ and (c) $100{ }^{\circ} \mathrm{C}$. 
In Table 2, the kinetic parameters resulting from these fittings are presented. The evident increase of the kinetic constants with the treatment temperature confirmed that non-enzymatic browning is favored by the increase in treatment temperature. In all, cases de $\mathrm{R}^{2}$ increasing with treatment temperature $\left(\mathrm{R}^{2} 0.982\right.$ for GAsn) at $120^{\circ} \mathrm{C}$. This indicated that this model fits the higher thermal treatment used.

Redness $\left(\mathrm{a}^{*}\right)$ and yellowness $\left(\mathrm{b}^{*}\right)$ color parameters were measured and plotted for all amino acids with glucose at different temperature. Curves of GGlu and GAsn proceed with the clockwise as well as heating time, as stated by Morales and Jiménez-Pérez (2001). The net increase in yellow-brown is observed during the first hours of heating reaching a maximum.

Table 2

Parameters of changes $\left(L^{*}\right)$ of color in the melanoidin model system

\begin{tabular}{lcccc}
\hline Melanoidins & $\begin{array}{c}\mathrm{T} \\
\left({ }^{\circ} \mathrm{C}\right)\end{array}$ & $K$ & $\begin{array}{c}k_{1} \times 10^{3} \\
\left(\mathrm{~min}^{-1}\right)\end{array}$ & $\mathrm{R}^{2}$ \\
\hline GAsn & 50 & 9.11 & 3.52 & 0.976 \\
& 85 & 18.62 & 1.22 & 0.973 \\
& 120 & 10.51 & 0.98 & 0.982 \\
\hline \multirow{2}{*}{ GAsp } & 50 & 37.82 & 4.06 & 0.938 \\
& 85 & 27.46 & 1.42 & 0.996 \\
& 120 & 19.42 & 2.13 & 0.998 \\
\hline \multirow{3}{*}{ GGlu } & 50 & 23.04 & 3.36 & 0.953 \\
& 85 & 24.81 & 3.37 & 0.895 \\
& 120 & 24.82 & 5.83 & 0.968 \\
\hline
\end{tabular}

Then curve of GAsn changing from yellow-brown to the brown-red zone showed a decrease in the $a^{*}$ and $b^{*}$ values with treatment time which is accentuated when the treatment temperature increases $\left(85{ }^{\circ} \mathrm{C}\right.$ and $120{ }^{\circ} \mathrm{C}$ ) (Figs. a and b). This reaction routes depending of the temperature and amino acid, decreased progressively. This indicates that there is an aggregation of particles of melanoidin with time at high temperature, showed in a previous work. However, The GGlu from the green-brown zone $b^{*}$ increased with the low temperature $\left(50{ }^{\circ} \mathrm{C}\right)$ (Fig. 5c) to yellow zone and decreased with high treatment in the red-brown. One the other hand the GAsp the $a^{*}$ was significantly reduced only at higher temperatures in the blue-red zone.
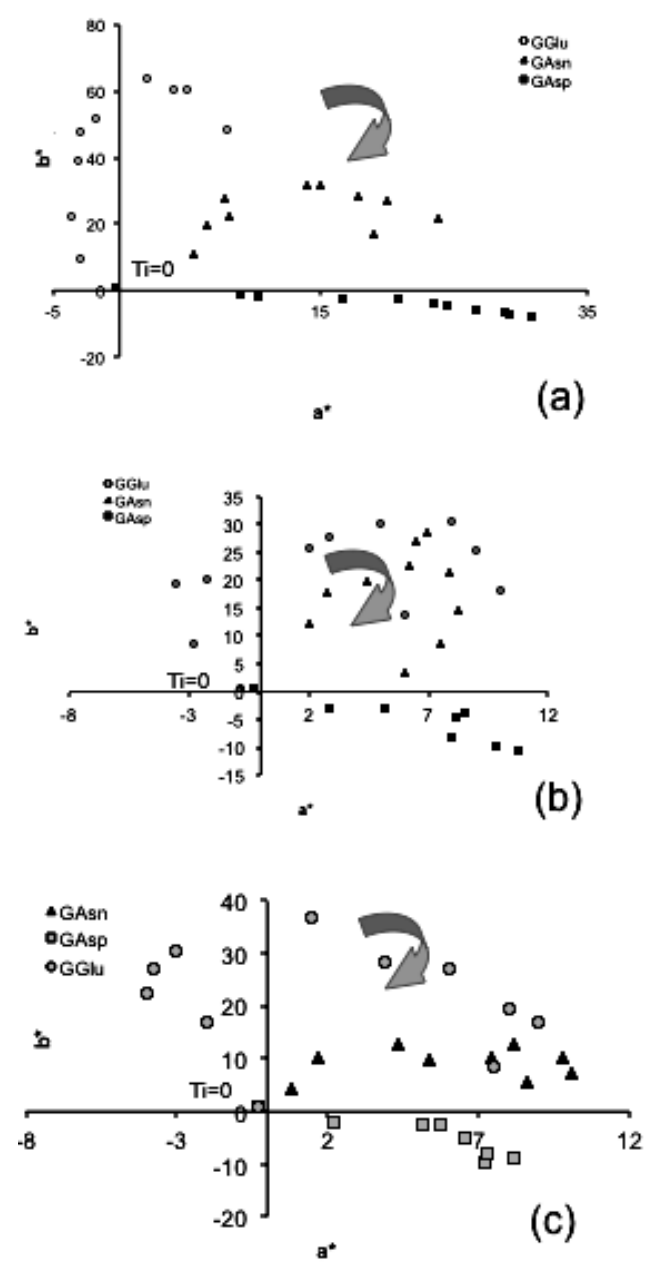

Figure 5. Changes in $b^{*}$ vs $a^{*}$ for the glucose and amino acid (Asn, Asp and Glu) model system during heating at different temperatures.

\section{Conclusions}

The main contribution of this paper is a complete kinetic assessment of the nonenzymatic browning of melanoidins from glucose and a single combination of amino acid under different temperature conditions, where the effects of temperature have been investigated. The selected amino acids, commonly found in apple juice and highly reactive in the Maillard reaction, were asparagine (Asn), aspartic acid (Asp) and glutamic acid (Glu). Modelling of first-order kinetic data revealed that the model was the most appropriate for 
describing color development in melanoidins concentrates. The processing temperature having different combination of amino acid had a strong impact on browning kinetics for melanoidins concentrates. It seems to have a significant effect on color development. Color of melanoidins formed in model systems decreased progressively with time. This indicates that there is an aggregation of particles of melanoidin with time at high temperature. The first quality impact by which consumers decide to acquire a product is its visual appearance. The color of products and various reactions, such as non-enzymatic browning (Maillard) reactions, can occur during the heating of fruit and vegetables and therefore affect their color for example juice processing. The retention of total color can be used as a quality indicator to evaluate the degree of deterioration due to thermal processing.

\section{Acknowledgements}

Echavarría A.P. wishes to thank the support of the Commission for Universities and Research of the DIUE in the Generalitat de Catalunya and the European Social Fund for financing this research.

\section{References}

Bharate, S.S.; Bharate, S.B. 2014. Non-enzymatic browning in citrus juice: chemical markers, their detection and ways to improve product quality 51(10): 2271-2288.

Billaud, C.; Maraschin, C.; Nicolas, J. 2004. Inhibition of polyphenoloxidase from apple by Maillard reaction products prepared from glucose or fructose with Lcysteine under various conditions of $\mathrm{pH}$ and temperature. LWT Food Science and Technology 37: 69-78.

Carabasa-Giribet, M.; Ibarz, A. 2000. Kinetics of colour development in aqueous fructose systems at high temperatures. Journal of the Science of Food and Agriculture 80(14): 2105-2113.

Contreras-Calderón, J.; Guerra-Hernández, E.; GarcíaVillanova, B. 2009. Utility of some indicators related to the Maillard browning reaction during processing of infant formulas. Food Chemistry 114: 1265-1270.

Demirhan, E.; Özbek, B. 2009. Color Change Kinetics of Microwave-Dried Basil. Drying Technology 27: 156166.

Echavarría, A.P.; Pagán, J.; Ibarz, A. 2013. Optimization of Maillard reaction products isolated from sugar-amino acid model system and their antioxidant activity. Afinidad 70(562): 86-92.
Echavarría, A.P.; Pagán, J.; Ibarz, A. 2012. Melanoidins Formed by Maillard Reaction in Food and Their Biological Activity. Food Engineering Review 4: 203 223.

Echavarría, A.P.; Torras, C.; Pagán, J.; Ibarz, A. 2011. Fruit juice processing and membrane technology application. Food Engineering Review 3: 136-158

Ganjloo, A.; Rahman, R.; Bakar, J.; Osman, A.; Bimakr, M. 2009. Modelling the kinetics of peroxidase inactivation and colour changes of seedless guava (Psidium guajava $\mathrm{L}$ ) during thermal treatments. World Applied Sciences Journal 7 (1): 105-112.

Gu, F.; Kim, J.; Abbas, S.; Zhang. X.; Xia, S.; Chen, Z. 2010. Structure and antioxidant activity of high molecular weight Maillard reaction products from casein-glucose. Food Chemistry 120: 505-511.

Ibarz, A.; Garza S.; Pagán, J. 2008. Inhibitory effect of melanoidins from glucose-asparagine on carboxypeptidases activity. European Food Research and Technology 226: 1277-1282.

Ibarz, A.; Pagán, J.; Garza, S. 2000. Kinetic models of nonenzymatic browning in apple puree. Journal of the Science of Food and Agriculture 80: 1162-1168.

Jaeger, H.; Janositz A.; Knorr, D. 2010. The Maillard reaction and its control during food processing. The potential of emerging technologies. Pathologie Biologie 58(3): 207-13.

Kim, J.; Lee, Y. 2008. The Influence of $\mathrm{pH}$ on the color development of melanoidins formed from fructose/amino acid enantiomer model Systems. Journal of Food science and nutrition 13: 306-312.

Kim, J.; Ra, K.; Suh, H. 2004. Hydrolysis of onion and kinetics of non-enzymatic browning of its hydrolysate. International Journal of Food Science \& Technology 10(1): 41-44.

Morales, F.; Jiménez-Pérez, S. 2001. Free radical scavenging of Maillard reaction products as related to color and fluorescence. Food Chemistry 72: 119-125.

Rattanathanalerk, M.; Chiewchan, N.; Srichumpoung, W. 2005. Effect of thermal processing on the quality loss of pineapple juice. Journal of Food Engineering 66: 259-265.

Rufián-Henares, J.; Morales, F. 2007. Functional properties of melanoidins: In vitro antioxidant, antimicrobial and antihypertensive activities. Food Research International 40(8): 995-1002.

Vaikousi, H.; Koutsoumanis, K.; Biliaderis, C. 2008. Kinetic modelling of non-enzymatic browning of apple juice concentrates differing in water activity under isothermal and dynamic heating conditions. Food Chemistry 107: 785-796.

Valdramidis, V.; Cullen, P.; Tiwari, B.; O’donnell, C. 2010. Quantitative modelling approaches for ascorbic acid degradation and non-enzymatic browning of orange juice during ultrasound processing. Journal of Food Engineering 96(3): 449-45

Zyzelewicz, D.; Kryslak, W.; Nebesny, E.; Budryn, G. 2014. Application of various methods for determination of the color of cocoa beans roasted under variable process parameter European Food Research and Technology 238: 549-563. 